\title{
A MATHEMATICAL MODEL OF TRAFFIC FLOW ON A NETWORK OF UNIDIRECTIONAL ROADS
}

\author{
Helge Holden, Nils Henrik Risebro
}

\begin{abstract}
We introduce a model that describes heavy traffic on a network of unidirectional roads. The model consists of a system of initial-boundary value problems for nonlinear conservation laws. We formulate and solve uniquely the Riemann problem for such a system and based on this, we then show existence of a solution to the Cauchy problem.
\end{abstract}

\begin{abstract}
A new problem, which has arisen in the twentieth century, is how to organize road traffic so that the full benefits of our increased mobility can be enjoyed at the lowest cost in human life and capital. The problem has many sides - constructional, legal, educational, administrative. - M.J. LIGHTHILL, G.B. WHITHAM

0. Introduction. The modelling of traffic flow by conservation laws is due to Lighthill and Whitham [15], [16], and Richards [19]. They argued as follows:

Consider a road with one lane with heavy traffic so that a continuum description is a good approximation. Let $\rho=\rho(x, t)$ denote the density of cars. Then conservation of the number of cars yields [14], [21] ('traffic hydrodynamics')

$$
\rho_{t}+(v \rho)_{x}=0
$$

where $v=v(x, t)$ denotes the velocity of cars at $(x, t)$.

Eq. (0.1) is valid for continuum descriptions of any conserved quantity. The first fundamental additional assumption we will make for the problem of traffic flow, is that the velocity field, $v$, only is a function of the density $\rho, v=v(\rho)$.

In addition it is reasonable to assume certain properties of the function $v$. When the density is small, there is a maximum velocity, $v_{\max }$, and when $\rho$ increases to some maximum capacity $\rho_{\max }$ the velocity vanishes.

A linear interpolation then gives the simplest possible velocity

$$
v=v_{\max }\left(1-\frac{\rho}{\rho_{\max }}\right) .
$$

One of the earliest velocity fields to be studied was the so-called Greenberg model with

$$
v(\rho)=v_{\max } \ln \left(\frac{\rho_{\max }}{\rho}\right)
$$

1980 Mathematics Subject Classification (1985 Revision). 35L65.

Key words and phrases. Traffic flow, conservation laws, network. 
supported by experimental data from the Lincoln tunnel in New York [6]. Other common models include ('Underwood's model')

$$
v=v_{\max } e^{\left(-\rho / \rho_{\max }\right)}
$$

and ('Greenshield's model')

$$
v=v_{\max }\left(1-\left(\frac{\rho}{\rho_{\max }}\right)^{n}\right)
$$

and ('the California model')

$$
v=v_{0}\left(\frac{1}{\rho}-\frac{1}{\rho_{\max }}\right)
$$

See $[17$, p. $296 \mathrm{ff}]$, and $[5$, p. $69 \mathrm{ff}]$ for an extensive comparison of these and other models and their experimental support. The resulting conservation law reads

$$
\rho_{t}+f(\rho)_{x}=0
$$

where

$$
f(\rho)=\rho v(\rho) .
$$

Given intial data of the form $\rho(x, 0)=\rho_{0}(x)$, the equation (0.7) can be analyzed using the comprehensive theory available for conservation laws [21], [20], [14].

We will use a flux function $f$ which is concave with a unique maximum, which is commonly assumed in traffic analysis, cf. [17], [5], [13]. Before we discuss various extensions and alternative approaches to the analysis presented here, we will give a brief summary of the content of this paper.

Consider any finite, connected directed graph. Let the edges model the roads with traffic in the given direction. The vertices correspond to junctions. In addition we may attach to the graph roads extending to infinity and connected to the graph at only one end. This system we call a network. The graph does not have to be planar, a non planar graph corresponds to the occurence of bridges or tunnels. On each individual road we assume that we have heavy traffic in one direction only, so that the density satisfies a conservation law (0.7) for a certain flux function $f$. The roads connecting two junctions have finite length. If there is not much interaction between the traffic in the two directions on a two-way road, or between the lanes in a multi lane road, we can approximate this by two or several unidirectional roads connecting the same two endpoints (junctions). 


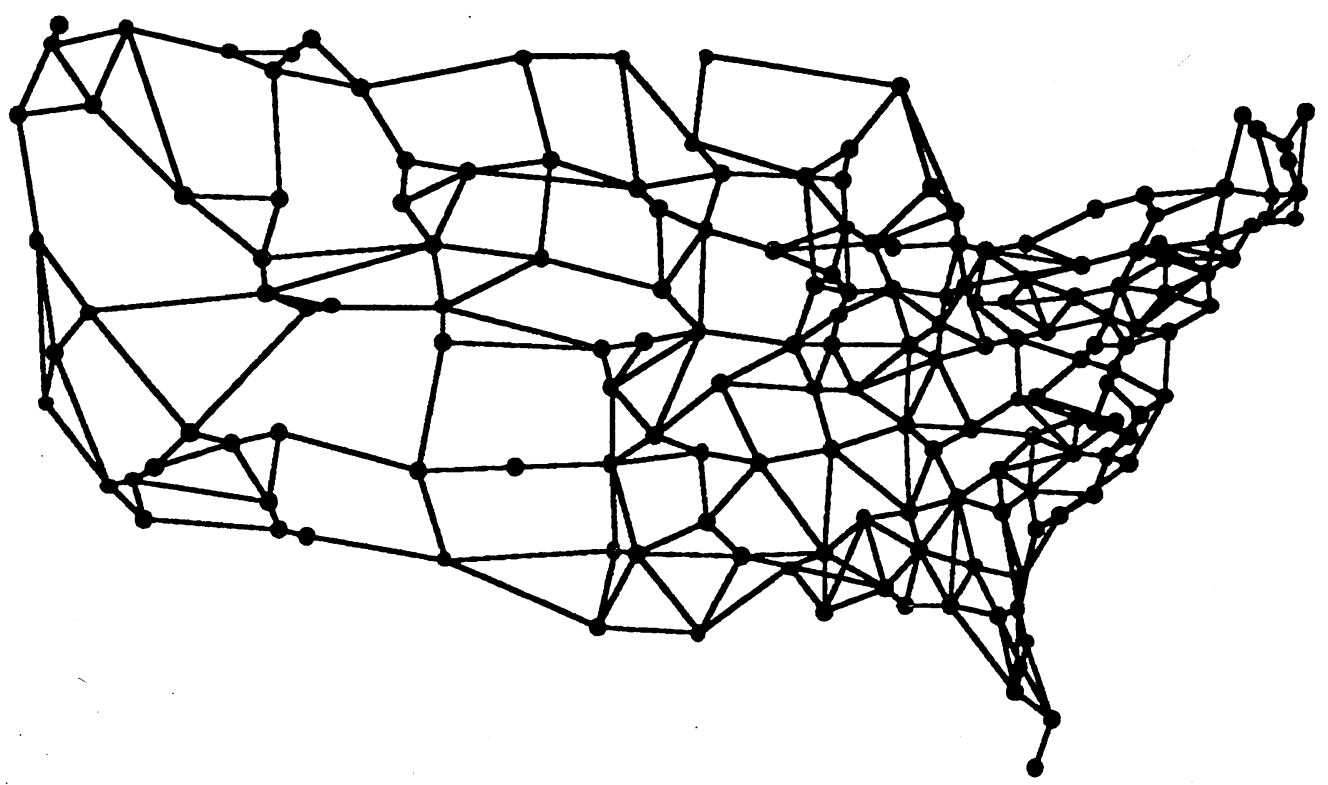

A TRAFFIC NETWORK.

A direct analysis of two-way traffic is complicated, see Bick and Newell [2], as this leads to a system of conservation laws. Even in the simplest cases one obtains a mixed hyperbolic/elliptic system for which even the solution of the Riemann problem is difficult, see e.g. [7], [10].

One has to specify how the cars distribute at the junctions in order to obtain a welldefined solution. We call this condition an entropy condition, in accordance with the terminology used for conservation laws. In this paper we will discuss an entropy condition that comes from maximizing the flux at each intersection. More precisely, given a concave function $g$ we maximize the flow at the junction $J$ measured by $\sum_{\text {roads } j \text { at } J} g\left(f\left(\rho_{j}\right) / f_{\text {max }}\right)$ where $f_{\max }$ denotes the maximum of $f$.

The solution of a conservation law develops singularities in finite time even for smooth initial data, thereby making it necessary to consider weak solutions of the partial differential equation.

Singularities in the sense of jump discontinuities moving with (finite) speed are called shocks. Specializing to Riemann initial data, i.e. two constant states separated by a single jump, see (2.1), the solution consists of combinations of two elementary waves; shock waves and rarefaction waves. (This terminology comes from gas dynamics.) Rarefaction waves are smooth solutions.

One carefully has to distinguish between the concept of wave speed (e.g. the speed of a shock) and the speed of an individual car. In the context of traffic flow this can be exemplified as follows.

When a car on a road with heavy traffic has to reduce its speed due to e.g. a traffic light turning red, we will have a shock wave, i.e. an abrupt change in density, propagating in the opposite direction of the individual cars. Conversely, as the red light turns green, we will have rarefaction wave extending in both directions as the cars in front accelerate and spread out and the density of cars decreases continuously.

The method we use to solve the initial-boundary value problem for the resulting system 
of conservation laws is based on the technique introduced by Dafermos [3], where one replaces the flux function by a polygon, i.e. a continuous piecewise linear approximation. In addition one approximates the initial value function by a step function, thereby obtaining (multiple) Riemann initial data. Since the flux function is piecewise linear, one obtains no rarefaction waves in the solution as these are approximated by small shocks. These shocks are then tracked. Dafermos' method was proved to converge in [9], [8].

Thus we first prove that the Riemann problems have a unique weak solution. The general Cauchy problem is approximated by finitely many Riemann problems and using estimates from [8] we can prove existence of a solution of the general Cauchy problem.

The general framework presented in this paper allows for many extensions. We have assumed the flux functions to be identical for all the roads in the network. One could allow for individual flux functions for each road, thereby studying e.g. how the quality of individual roads, as measured by the flux function, influence traffic on the network. Furthermore one could study the situation where the flux function also depends on position on the road, i.e. $f=f(x, \rho)$, see e.g. [16], [4], [18], which could be used as a model of bottlenecks. In addition traffic lights could be built into the model, see [16], [21].

Recently one has started to study stochastic conservation laws, see [11], [12]. In this context that would correspond to the situation where e.g. the initial density in not known exactly or the exact form of $f$ is uncertain. The analysis in this paper could be extended to include this.

For extensive discussions of the advantages and disadvantages of the hydrodynamical approach to traffic flow we refer to [17], [5], [13], which also contain alternative models for traffic flow.

1. The Riemann problem. Consider a network of unidirectional roads which are connected at intersections. The precise definition of network reads

Definition 1.1. By a network we mean a finite, connected directed graph where we in addition may attach a finite number of directed curves extending to infinity.

Let each edge or curve correspond to a road, and assume that the traffic is in the direction given by the direction in the network. The vertices correspond to junctions or intersections. A road may be connected to other roads at both ends or at one end only, in the latter case the unconnected end is assumed to extend to infinity. We assume that we have have $N$ roads which are connected at $M$ junctions. 


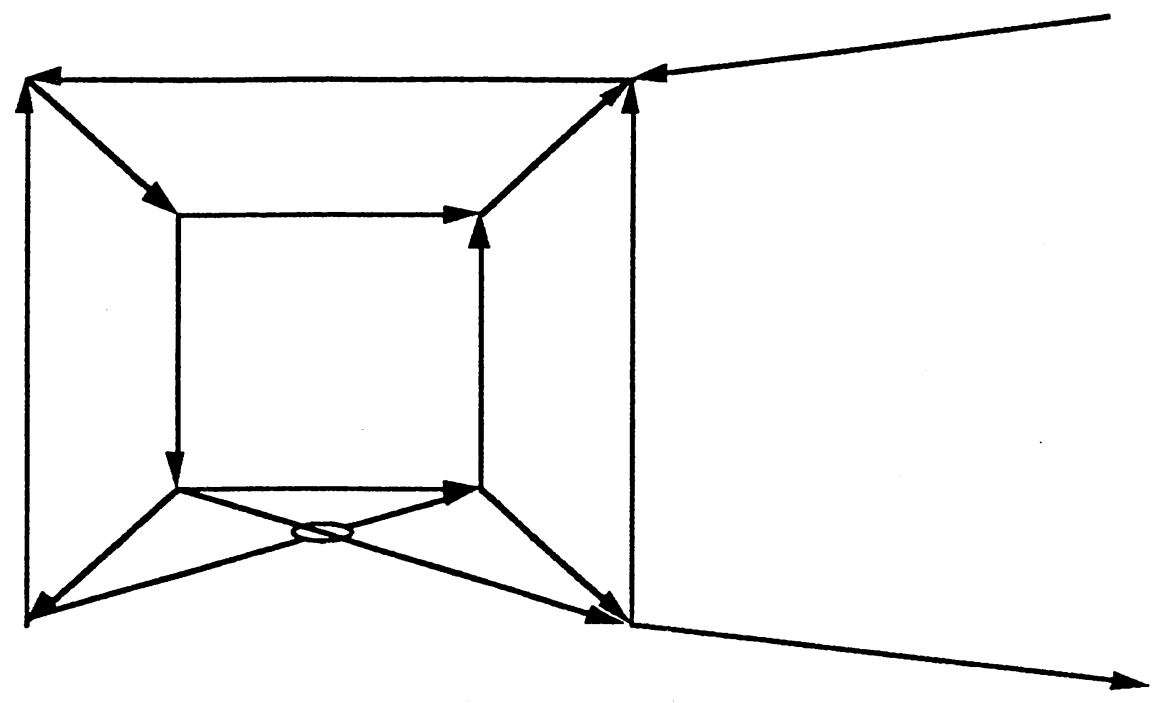

A NETWORK.

Let $\rho_{i}=\rho_{i}(x, t)$ denote the density of cars at road $i$ with $x \in\left[a_{i}, b_{i}\right]$ where $a_{i}=-\infty$ or $b_{i}=\infty$ if the road extends to infinity. Assume that the traffic goes in direction from $a_{i}$ to $b_{i}$. We will use the notation

$$
\hat{\rho}\left(x_{1}, \ldots, x_{N}, t\right)=\left(\rho_{1}\left(x_{1}, t\right), \ldots, \rho_{N}\left(x_{N}, t\right)\right), \quad t \geq 0, \quad x_{i} \in\left[a_{i}, b_{i}\right], \quad i=1, \ldots, N,
$$

which should be interpreted in the distributional sense. Away from intersections, conservation of cars yields [21], [14]

$$
\frac{\partial \rho_{i}}{\partial t}+\frac{\partial f\left(\rho_{i}\right)}{\partial x}=0, \quad i=1, \ldots, N .
$$

At each road we have given an initial density, viz.

$$
\rho_{i}(x, 0)=\rho_{i, 0}(x), \quad x \in\left[a_{i}, b_{i}\right], \quad i=1, \ldots, N .
$$

The roads will be coupled in terms of boundary conditions at the intersections. The weak formulation of the system of conservation laws will provide us with the right boundary conditions.

Consider a junction $J$, and assume, by relabeling if necessary, that the roads $1, \ldots, n$ enter $J$ and roads $n+1, \ldots, n+m$ leave $J$. (Whenever we study a fixed intersection $J$ in the following we will assume this labeling.) Write

$$
c_{i}= \begin{cases}b_{i}, & \text { for } i=1, \ldots, n \\ a_{i}, & \text { for } i=n+1, \ldots, n+m .\end{cases}
$$

Let $\phi=\left\{\phi_{i}\right\}_{1}^{N}$ be smooth test functions with compact support that also are $C^{1}$ smooth across junctions, i.e.,

$$
\phi_{i}\left(c_{i}, t\right)=\phi_{j}\left(c_{j}, t\right) \quad \text { and } \quad \frac{\partial \phi_{i}}{\partial x}\left(c_{i}, t\right)=\frac{\partial \phi_{j}}{\partial x}\left(c_{j}, t\right)
$$


for all $1 \leq i, j \leq n+m$, and similarly for other junctions. A weak solution of (1.2) is a set of functions $\rho_{i}(x, t)$ which satisfies

$$
\sum_{i=1}^{N}\left(\int_{0}^{\infty} \int_{a_{i}}^{b_{i}}\left[\rho_{i} \frac{\partial \phi_{i}}{\partial t}+f\left(\rho_{i}\right) \frac{\partial \phi_{i}}{\partial x}\right] d x d t+\int_{a_{i}}^{b_{i}} \rho_{i, 0}(x) \phi_{i}(x, 0) d x\right)=0
$$

for all $\phi$ satisfying (1.5).

By performing an analysis similar to the derivation of the Rankine-Hugoniot jump relation for shocks (see e.g. [20, p. 246ff]) one easily obtains, as a consequence of the weak formulation, that for each intersection $J$

$$
\sum_{i=1}^{n} f\left(\rho_{i}\left(b_{i}, \cdot\right)\right)=\sum_{i=n+1}^{n+m} f\left(\rho_{i}\left(a_{i}, \cdot\right)\right)
$$

for $t>0$, i.e., all cars that enter an intersection must emerge at some other road leaving the intersection. We call (1.7) the Rankine-Hugoniot condition for intersections. (Eq. (1.7) resembles Kirchhoff's law in electromagnetism, but we will not use that terminology here) Alternatively we could consider the system (1.2) of $N$ separate equations and impose boundary conditions (1.7) at each junction.

We will make certain assumptions on the function $f$ that are reasonable in the context of traffic flow. Normalize $\rho$ so that $\rho_{\max }=1$. We essentially assume that $f$ has a unique maximum, viz.

$$
f(0)=f(1)=0, \quad \exists \sigma \in\langle 0,1\rangle: f^{\prime}(\sigma)=0, \quad(\rho-\sigma) f^{\prime}(\rho)<0, \quad \rho \neq \sigma .
$$

These restrictions hold for flux functions commonly used in the analysis of traffic flow. In the remaining part of this section we study of the Riemann problem for the single junction $J$.

Definition 1.2. By the weak solution of the Riemann problem for the junction $J$ we mean the weak solution of the initial value problem (1.2), (1.3), (1.7) for the network consisting of the single junction $J$ with $n$ incoming roads and $m$ outgoing roads, all extending to infinity. The initial data are given by

$$
\rho_{i, 0}(x)=\rho_{i, 0}, \quad x \in\left[a_{i}, b_{i}\right], \quad i=1, \ldots, n+m,
$$

where $\rho_{i, 0}, i=1, \ldots, n+m$ are constants.

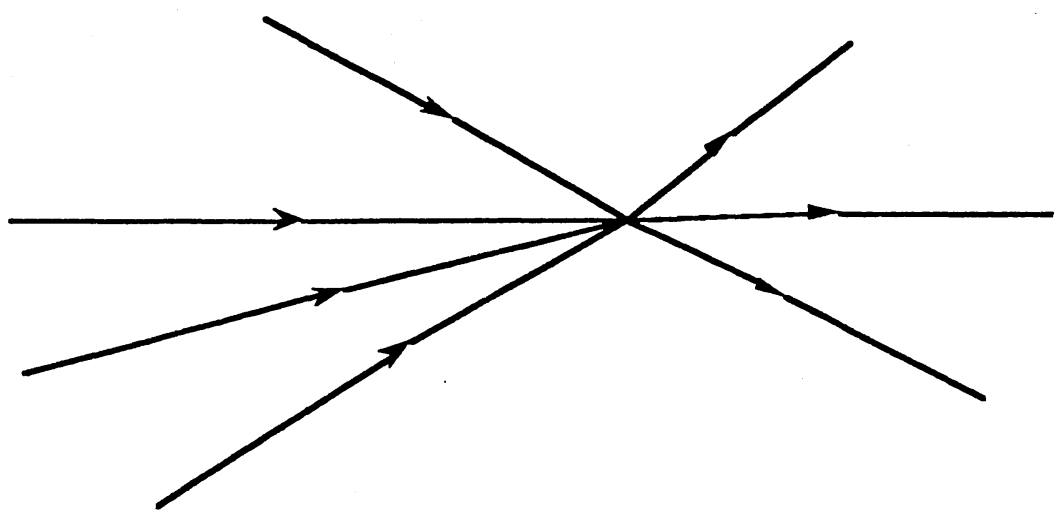

The RiemanN PROBLEM. 
We now turn to the discussion of the entropy condition. Consider once more the junction $J$ of $n$ incoming and $m$ outgoing roads. We write the solution at the junction as

$$
\bar{\rho}=\left(\bar{\rho}_{1}, \bar{\rho}_{2}\right) \in \mathbb{R}^{n+m}
$$

where

$$
\begin{aligned}
& \bar{\rho}_{1}=\left(\rho_{1}\left(c_{1}, \cdot\right), \ldots, \rho_{n}\left(c_{n}, \cdot\right)\right) \\
& \bar{\rho}_{2}=\left(\rho_{n+1}\left(c_{n+1}, \cdot\right), \ldots, \rho_{n+m}\left(c_{n+m}, \cdot\right)\right) .
\end{aligned}
$$

The set of all possible densities where the Rankine-Hugoniot condition for $J$ holds is

$$
H_{J}=\left\{\bar{\rho}=\left(\bar{\rho}_{1}, \bar{\rho}_{2}\right) \in \mathbb{R}^{n+m} \mid \sum_{i=1}^{n} f\left(\rho_{i}\left(b_{i}, \cdot\right)\right)=\sum_{i=n+1}^{n+m} f\left(\rho_{i}\left(a_{i}, \cdot\right)\right)\right\} .
$$

At each intersection we assume that there is a tendency for the total flux across each junction to approach some predefined value. This could be the maximum possible flux across the junction or some other, perhaps observed, value of the flux. We express this mathematically by an additional boundary condition which we call an entropy condition. Let the flux function $f$ satisfy $(\mathcal{F})$, and denote $f_{\max }=f(\sigma)$. Let $g$ be some differentiable strictly concave function of a single variable defined on $\mathbb{R}$. We define the entropy of the junction $J$ as

$$
E_{J}=\sum_{i=1}^{n+m} g\left(\frac{f_{i}}{f_{\max }}\right), \quad f_{i}=f\left(\rho_{i}\left(c_{i}\right)\right) .
$$

The entropy condition reads:

Find $\bar{\rho}=\left(\rho_{1}, \ldots, \rho_{n+m}\right) \in H_{J}$ which maximizes $E_{J}$, such that $\rho_{i} \in[\sigma, 1]$, for $i=1, \ldots, n$, and $\rho_{i} \in[0, \sigma]$ for $i=n+1, \ldots, n+m$.

Observe that for such $\bar{\rho}$ there is a one-to-one correspondence between $\rho_{i}$ and $f_{i}=f\left(\rho_{i}\right)$. Denote $\phi=\frac{f}{f_{\max }}$ and let

$$
\Psi(\rho)= \begin{cases}(\phi(\rho),-1) & \text { if } \rho \leq \sigma \\ (\phi(\rho), 1) & \text { if } \rho>\sigma .\end{cases}
$$

The Hugoniot relation (1.7) now reads

$$
\sum_{i=1}^{n} \phi_{i}=\sum_{i=n+1}^{n+m} \phi_{i}
$$

Consider a junction $J$ with roads $1, \ldots, n$ incoming and $n+1, \ldots, n+m$ outgoing. To simplify the notation we assume that $c_{1}=\cdots=c_{n+m}=0$ and write

$$
\begin{aligned}
& \hat{\rho}(x, 0)=\left(\rho_{1,0}, \ldots, \rho_{n+m, 0}\right)=\left(\rho_{1}^{L}, \ldots, \rho_{n}^{L}, \rho_{n+1}^{R}, \ldots, \rho_{n+m}^{R}\right) \\
& \hat{\rho}(0, t)=\left(\rho_{1}, \ldots, \rho_{n+m}\right) .
\end{aligned}
$$


Define

$$
\rho_{i}^{\min }=\min \left\{\rho \in[\sigma, 1] \mid f(\rho)=f\left(\rho_{i}^{L}\right)\right\}, \quad \rho_{i}^{\max }=\max \left\{\rho \in[0, \sigma] \mid f(\rho)=f\left(\rho_{i}^{R}\right)\right\}
$$

Then

$$
\begin{aligned}
& \rho_{i} \in\left\{\rho_{i}^{L}\right\} \cup[\sigma, 1], \quad i=1, \ldots, n \\
& \rho_{i} \in\left\{\rho_{i}^{R}\right\} \cup[0, \sigma], \quad i=n+1, \ldots, n+m,
\end{aligned}
$$

because any wave emerging from an intersection along some road must have positive speed if the road emerges from the intersection and negative speed if the road enters the intersection, cf. figure below.

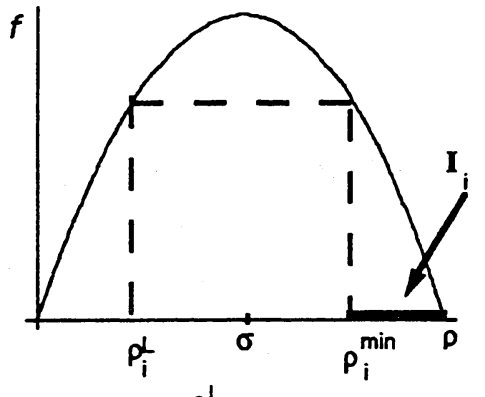

$p_{i}^{L}<\sigma$

$i=1, \ldots, n$

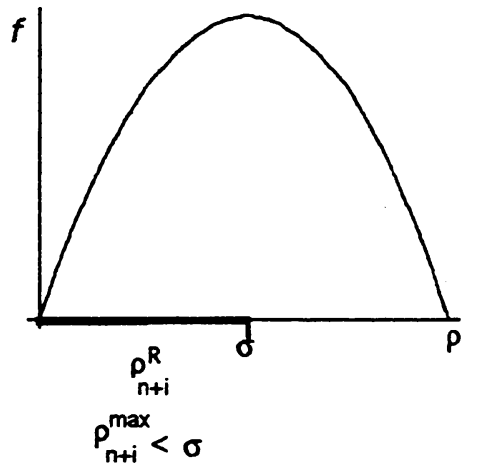

$i=1, \ldots, m$

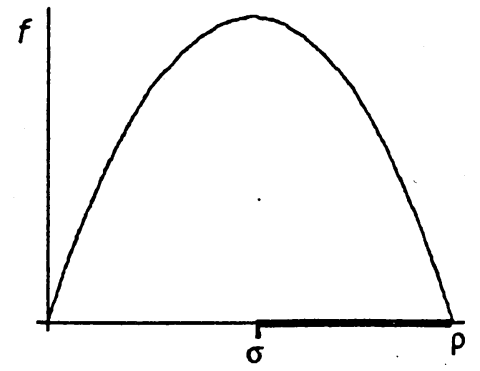

$P_{i}^{L}>\sigma$

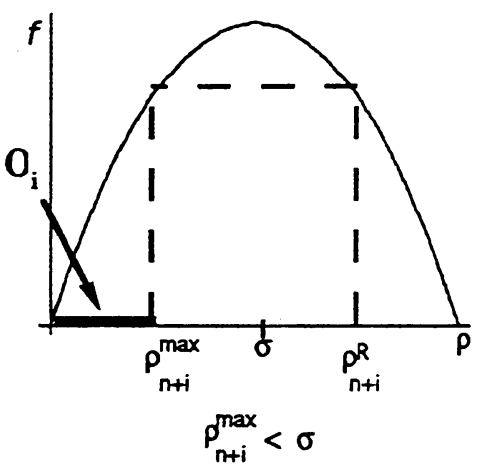

$\rho_{n+i}^{\max }<\sigma$

Define

$$
\begin{aligned}
I_{i} & =\left[\rho_{i}^{\min }, 1\right] \subset[\sigma, 1] \quad i=1, \ldots, n \\
O_{i} & =\left[0, \rho_{i}^{\max }\right] \subset[0, \sigma], \quad i=n+1, \ldots, n+m
\end{aligned}
$$

and

$$
I=\prod_{i=1}^{n} I_{i}, \quad O=\prod_{i=n+1}^{n+m} O_{i} \quad \text { and } \quad A=\prod_{i=1}^{n}[\sigma, 1] \times \prod_{i=n+1}^{n+m}[0, \sigma] \subset \mathbb{R}^{n+m}
$$


We may assume that the solution at the intersection $J$ is in $A$ from the following argument. If $\rho_{i}=\rho_{i}(x, t), i \leq n$ satisfies the Rankine-Hugoniot condition (1.7), then so will

$$
\tilde{\rho}_{i}(x, t)=\left\{\begin{array}{l}
\rho_{i}^{\min } \text { if } x=0 \\
\rho_{i}^{L} \text { if } x<0
\end{array}\right.
$$

which is identical to $\rho_{i}$ in $L_{1}$. In this case we call the shock with zero speed at $x=0$ a virtual shock. Similarly, we may redefine $\rho_{i}, i \geq n$ at the origin using $\rho_{i}^{\max }$ without changing the solution in $L_{1}$. From speed compatibility conditions some of the $\phi_{i}$ may be restricted to certain intervals $\left[0, \psi_{i}\right]$. Consider for the moment the entropy function $E_{J}$ as a function of $\phi_{1}, \ldots, \phi_{n+m} \in[0,1]$, and replace $\phi_{n+m}$ in $E_{J}$ using (1.14). Thus

$$
E_{J}=\sum_{i=1}^{n+m-1} g\left(\phi_{i}\right)+g\left(\sum_{i=1}^{n} \phi_{i}-\sum_{i=n+1}^{n+m-1} \phi_{i}\right)
$$

and

$$
\frac{\partial E_{J}}{\partial \phi_{i}}=g^{\prime}\left(\phi_{i}\right)+\frac{\partial \phi_{n+m}}{\partial \phi_{i}} g^{\prime}\left(\phi_{n+m}\right)
$$

where

$$
\frac{\partial \phi_{n+m}}{\partial \phi_{i}}= \begin{cases}+1 & \text { for } i=1, \ldots, n \\ -1 & \text { for } i=n+1, \ldots, n+m-1 .\end{cases}
$$

Therefore $\nabla E_{J}=0$ implies that $g^{\prime}\left(\phi_{i}\right)=-g^{\prime}\left(\phi_{n+m}\right)$ for $i=1, \ldots, n$, and $g^{\prime}\left(\phi_{i}\right)=g^{\prime}\left(\phi_{n+m}\right)$ for $i=n+1, \ldots, n+m-1$. Thus

$$
\phi_{i}^{\mathcal{G}}=\phi_{i}= \begin{cases}\frac{m}{n} \phi_{m+n} & \text { for } i=1, \ldots, n \\ \phi_{m+n} & \text { for } i=n+1, \ldots, n+m-1,\end{cases}
$$

where $\phi_{m+n}$ is the solution of the equation

$$
g^{\prime}\left(\frac{m}{n} \phi_{m+n}\right)=-g^{\prime}\left(\phi_{m+n}\right)
$$

provided this equation has a solution $\phi_{n+m} \in[0,1]$. This solution is always a maximum for $E_{J}$ since $E_{J}\left(\phi_{1}, \ldots, \phi_{n+m-1}\right)$ is a concave function. A unique solution will exist in [0,1] if e.g. $g^{\prime}(0)=-g^{\prime}(1)$. If all the $\phi_{i}^{\mathcal{E}}$ found by (1.25) are such that $\phi_{i}<\psi_{i}$ this will be the unique entropy solution. Assume that $\phi_{i}>\psi_{i}$ for $i=k_{1}, \ldots, k_{\ell}$. If $\ell=n+m$, then the entropy solution is $\phi_{i}^{\mathcal{E}}=\psi_{i}, i=1, \ldots, n+m$. If $\ell<n+m$, we assume that $\phi_{n+m}<\psi_{n+m}$. In this case let

$$
c=\sum_{k_{j} \leq n} \psi_{k_{j}}-\sum_{k_{j}>n} \psi_{k_{j}}
$$


A similar calculation as presented above gives

$$
\phi_{i}= \begin{cases}\frac{\bar{m} \phi_{m+n}-c}{\bar{n}} & \text { for } i=1, \ldots, n, i \neq k_{j} \\ \phi_{m+n} & \text { for } i=n+1, \ldots, n+m-1, i \neq k_{j},\end{cases}
$$

where $\tilde{n}$ is the number of incoming roads which not in the set $\left\{k_{j}\right\}$, similarly for $\tilde{m}$. Now $\phi_{m+n}$ is the solution of the equation

$$
g^{\prime}\left(\frac{\tilde{m} \phi_{m+n}-c}{\tilde{n}}\right)=-g^{\prime}\left(\phi_{n+m}\right) \text {. }
$$

Let $\phi_{i}^{\mathcal{E}}$ denote the entropy solution we have found. We have now proved the following theorem.

Theorem 1.1. Assume that $f$ satisfies $(\mathcal{F})$, and let $g$ be a strictly concave differentiable function with $g^{\prime}(0)=-g^{\prime}(1)$. Then the Riemann problem for the single junction $J$ with entropy condition $(\mathcal{E})$ has a unique solution $\bar{\phi}^{\mathcal{E}}$.

2. Construction of approximate solutions. We will now turn our attention to the general Cauchy problem with the entropy condition $(\mathcal{E})$ imposed on each intersection. Based on Dafermos' method [3], we will construct approximate solutions to this problem, and in the next section we will show that this construction yields a convergent subsequence.

First we briefly review the solution of the Riemann problem for the scalar conservation law if the flux function $f$ is piecewise linear. Consider the Riemann problem

$$
\begin{gathered}
u_{t}+f(u)_{x}=0 \\
u(x, 0)=\left\{\begin{array}{l}
u_{\ell} \text { for } x<0 \\
u_{r} \text { for } x \geq 0,
\end{array}\right.
\end{gathered}
$$

where $u$ is a scalar function. Let $u_{0}=u_{\ell}$ and $u_{N}=u_{r}$ and

$$
-\infty=u_{-1}<u_{0}<u_{1}<\cdots<u_{N}<u_{N+1}=\infty
$$

and

$$
f_{i}=f\left(u_{i}\right) \in \mathbb{R}, \quad i=0, \ldots, N
$$

and assume that $f$ is piecewise linear, i.e.,

$$
u \in\left[u_{i}, u_{i+1}\right] \Rightarrow f(u)=\frac{f_{i+1}-f_{i}}{u_{i+1}-u_{i}}\left(u-u_{i}\right)+f_{i}, \quad i=0, \ldots, N-1
$$

and

$$
u \leq u_{0} \Rightarrow f(u)=f\left(u_{0}\right), \quad u \geq u_{N} \Rightarrow f(u)=f\left(u_{N}\right) .
$$

Let $f_{c}$ denote the lower convex envelope of $f$ on $\left[u_{\ell}, u_{\tau}\right]$. Then also $f_{c}$ is piecewise linear. Let

$$
-\infty=\tilde{u}_{-1}<\tilde{u}_{0}<\tilde{u}_{1}<\cdots<\tilde{u}_{M}<\tilde{u}_{M+1}=\infty
$$

with

$$
\tilde{u}_{0}=u_{0}, \quad \tilde{u}_{M}=u_{N}, \quad \tilde{f}_{i}=f_{c}\left(\tilde{u}_{i}\right)
$$

and

$$
\left\{\tilde{u}_{0}, \ldots, \tilde{u}_{M}\right\} \subseteq\left\{u_{1}, \ldots, u_{N}\right\}
$$

and such that $f_{c}$ is continuous and linear on each interval $\left[u_{i}, u_{i+1}\right]$. Then we have that [1] 
Theorem 2.1. Let $f$ be given by (2.4) and define its lower convex envelope on $\left[u_{\ell}, u_{r}\right]$ by $f_{c}$. Then the solution of (2.1) is given by

$$
u(x, t)= \begin{cases}u_{\ell}, & \text { for } x<\tilde{s}_{0} t \\ \tilde{u}_{i}, & \text { for } \tilde{s}_{i-1} t<x<\tilde{s}_{i} t, \quad i=1, \ldots, M \\ u_{\tau}, & \text { for } x>\tilde{s}_{M+1} t\end{cases}
$$

where

$$
\tilde{s}_{i}=\frac{\tilde{f}_{i+1}-\tilde{f}_{i}}{\tilde{u}_{i+1}-\tilde{u}_{i}}, \quad i=0, \ldots, M
$$

Observe that in this case $u(x, t)$ is a piecewise constant function in $x / t$. For a scalar conservation law on the line, Dafermos' scheme consists in approximating the initial function by a step function, thereby generating a series of Riemann problems. The solution of these will then define a set of discontinuities which propagate linearly in $(x, t)$ space. At some $t_{1}>0$ two of these discontinuities will collide, and one then solves the Riemann problem defined by the values to the right and left of the collision. This gives another set of discontinuities and the process can be continued up to the next collision time. In [9] it is shown that this is a well-defined process and that the solutions generated converge as the approximation of the flux function $f$ and the initial value function $u_{0}$ converge.

We will use this strategy to construct an approximation to the solution of (1.2), (1.3). First we make a polygonal approximation of $f$. Let $k$ be some positive even integer and divide the interval $[0, \sigma]$ into $k / 2+1$ intervals of length $2 \sigma / k$. The interval $\langle\sigma, 1]$ is then divided at points $\left\{\tilde{\rho}_{j}\right\}_{j=k / 2+2}^{k}$ chosen such that

$$
f\left(2 \frac{j}{k} \sigma\right)=f\left(\tilde{\rho}_{k-j}\right)
$$

for $j=0, \ldots, k / 2$. For $j \leq k / 2$ we define $\tilde{\rho}_{j}=2 \frac{\dot{j}}{k} \sigma$. We define $f^{k}(\rho)$ to be the piecewise linear continuous function

$$
f^{k}(\rho)=f\left(\tilde{\rho}_{j}\right)+\frac{f\left(\tilde{\rho}_{j}\right)-f\left(\tilde{\rho}_{j+1}\right)}{\tilde{\rho}_{j}-\tilde{\rho}_{j+1}}\left(\rho-\tilde{\rho}_{j}\right) \quad \text { for } \quad \rho \in\left[\tilde{\rho}_{j}, \tilde{\rho}_{j+1}\right]
$$

Having approximated the flux function $f$ we now approximate the initial data $\rho_{i, 0}$ with a step function $\rho_{i, 0}^{k}(x)$ taking values in the set $\left\{\tilde{\rho}_{j}\right\}_{j=0}^{k+1}$. The solution of the Riemann problem (2.1) away from the intersections will take values in $\left\{\tilde{\rho}_{j}\right\}$ (cf. Theorem 2.1), but the solution of the Riemann problem (1.8) at the junctions may take us outside $\left\{\tilde{\rho}_{j}\right\}$. However, we approximate the exact solution in the following way: Assume that on one particular road the exact solution of the Riemann problem adjacent to the junction is given by $\rho^{\prime} \in\left\langle\rho_{j}, \rho_{j+1}\right\rangle$ and let the initial density on that road problem be $\rho$. Then the approximate solution will be $\rho_{j}$ if $\left|\rho_{j}-\rho\right|<\left|\rho_{j+1}-\rho\right|$ and the approximate solution will be $\rho_{j+1}$ if this inequality does not hold. By doing this we introduce an error in conservation and 
the approximate solution is no longer an exact weak solution of an approximate problem. But this error is of size $O\left(\frac{N}{k}\right)$ and thus vanishes as $k \rightarrow \infty$.

For the Riemann problems defined by $f=f^{k}$ and the discontinuities of $\rho_{i, 0}^{k}$, we can find the solution using Theorem 2.1. The approximate solution $\hat{\rho}^{k}=\left(\rho_{1}^{k}, \ldots, \rho_{N}^{k}\right)$ defines a set of discontinuities moving in the intervals $\left[a_{i}, b_{i}\right]$ for $i=1, \ldots, N$. Clearly $\hat{\rho}^{k}$ can be defined at least until the first collision of discontinuities. This collision defines a new Riemann problem whose solution creates new discontinuites which can be propagated until the next collision and so on. Obviously, this can be repeated an arbitrary number of times. Below we will show that $\hat{\rho}^{k}$ can indeed be defined up to any time, but first we must show some lemmas.

We now have that $\rho_{i}^{k}(x, t), i=1, \ldots, N$ will be a step function in $x$ and thus defines a number of constant states $\rho_{i, j}^{k}$ for $j=0, \ldots, n_{i}$, such that $\rho_{i, 0}^{k}=\rho_{i}^{k}\left(a_{i}, \cdot\right)$ and so on. We will also label the times when some discontinuities collide, either with each other or with an intersection, by $t_{\ell}$ for $\ell=1,2, \ldots$

Lemma 2.1. Assume that we have an entropy state $\bar{\phi}$ which is a solution of the Riemann problem for the junction $J$. Consider a shock colliding with $J$ from road $k$. Let the new entropy solution after the collision be denoted by $\bar{\phi}^{\prime}$. If $k \leq n$ then

$$
\begin{array}{ll}
\phi_{i}^{\prime} \geq \phi_{i} & \text { for } i=1, \ldots, n, i \neq k, \\
\phi_{i}^{\prime} \leq \phi_{i} & \text { for } i=n+1, \ldots, n+m,
\end{array}
$$

and if $k>n$

$$
\begin{array}{ll}
\phi_{i}^{\prime} \leq \phi_{i} & \text { for } i=1, \ldots, n, \\
\phi_{i}^{\prime} \geq \phi_{i} & \text { for } i=n+1, \ldots, n+m, i \neq k
\end{array}
$$

Proof. We will show the lemma in case $k \leq n$, the proof in the other case is identical. Since the colliding discontinuity has positive speed, the density to the left of the discontinuity, $\tilde{\rho}$, must be in the set $\left\{\rho \mid \rho<\sigma\right.$ and $\left.\phi(\rho)<\phi_{k}\right\}$. The possible densities adjacent to the juntion on road $k$ after the collision are from the set $\{\rho \mid \rho>\sigma$ and $\phi(\rho) \leq \phi(\tilde{\rho})\}$. Now (2.13) will follow if

$$
\left.\frac{\partial E_{J}}{\partial \phi_{i}}\right|_{\left(\phi_{1}, \ldots, \phi_{k}^{\prime}, \ldots, \phi_{m+n-1}\right)} \begin{cases}>0 & \text { for } i=1, \ldots, n, i \neq k, \\ <0 & \text { for } i=n+1, \ldots, n+m .\end{cases}
$$

Let $\tilde{\phi}_{n+m}=\sum_{i \leq n, i \neq k} \phi_{i}-\sum_{i>n} \phi_{i}+\phi_{k}^{\prime}$. Since $\phi_{k}^{\prime}<\phi_{k}, \tilde{\phi}_{n+m}<\phi_{n+m}$, and since $g^{\prime}$ is a decreasing function $g^{\prime}\left(\tilde{\phi}_{n+m}\right)>g^{\prime}\left(\phi_{n+m}\right)$. Thus from (1.23) we get for $i \leq n$

$$
0=\left.\frac{\partial E_{J}}{\partial \phi_{i}}\right|_{\bar{\phi}}=g^{\prime}\left(\phi_{i}\right)+g^{\prime}\left(\phi_{n+m}\right)<g^{\prime}\left(\phi_{i}\right)+g^{\prime}\left(\tilde{\phi}_{n+m}\right)=\left.\frac{\partial E_{J}}{\partial \phi_{i}}\right|_{\left(\phi_{1}, \ldots, \phi_{k}^{\prime}, \ldots, \phi_{m+n-1}\right)} .
$$

Similarly for $i>n$

$$
\left.\frac{\partial E_{J}}{\partial \phi_{i}}\right|_{\left(\phi_{1}, \ldots, \phi_{k}, \ldots, \phi_{m+n-1}\right)}<0
$$

and the lemma is proved. 
Lemma 2.2. Assume that we have an entropy state $\bar{\phi}$ which is a solution of the Riemann problem for the junction $J$. Consider a shock colliding with $J$ from road $k$. Let the new entropy solution after the collision be denoted by $\bar{\phi}^{\prime}$. Then

$$
\left|\phi_{k}-\phi_{k}^{\prime}\right|=\sum_{i \neq k}\left|\phi_{i}-\phi_{i}^{\prime}\right|
$$

Proof. Without loss of generality we can assume that $k \leq n$. By the previous lemma $\phi_{i}^{\prime} \geq \phi_{i}$ for $i \leq n, i \neq k$, and $\phi_{i}^{\prime} \leq \phi_{i}$, subtracting the Rankine-Hugoniot relations for $\bar{\phi}$ and $\bar{\phi}^{\prime}$ we get

$$
\phi_{k}-\phi_{k}^{\prime}=\sum_{i \neq k}^{n}\left(\phi_{i}^{\prime}-\phi_{i}\right)-\sum_{i=n+1}^{n+m}\left(\phi_{i}^{\prime}-\phi_{i}\right)=\sum_{i \neq k}^{n}\left(\phi_{i}^{\prime}-\phi_{i}\right)+\sum_{i=n+1}^{n+m}\left(\phi_{i}-\phi_{i}^{\prime}\right) .
$$

All terms in the last equation are positive and the lemma follows.

For $t_{\ell-1}<t<t_{\ell}$ we define the functional $T^{\ell}$ by

$$
T^{\ell}=\sum_{r=1}^{N} T_{r}^{\ell}=\sum_{r=1}^{N} \sum_{i=1}^{n_{r}}\left|z_{r, i}^{k}-z_{r, i-1}^{k}\right|
$$

where $z=\Psi(\rho)$. We now have

Lemma 2.3.

$$
T^{\ell+1} \leq T^{\ell}
$$

Proof. It follows from Theorem 3.1 in [9] that $T$ does not increase when two discontinuities collide. In the case where a discontinuity collides with a junction Lemma 2.2 implies that $T$ does not increase. This is so since the waves emitted from a junction in the approximated solution always will be smaller or equal to the correct wawes described by Lemma 2.2 .

Now since $\phi$ takes values in a finite set and $T$ is positive it follows that after some finite number of collisions, $L, T$ remains equal to some constant $T^{L}$. If $T$ is constant, for collisions between discontinuities, we can only have collisions between discontinuities separating $\left(\phi_{l}, i_{l}\right)$ and $\left(\phi_{m}, i_{m}\right)$, and $\left(\phi_{m}, i_{m}\right)$ and $\left(\phi_{r}, i_{\tau}\right)$. In this case $\phi_{m}$ must be between $\phi_{l}$ and $\phi_{r}$. In particular, this implies that both discontinuities have positive or negative speed. So after $t_{L}$, all roads must have discontinuities of either positive or negative speed only. Let us examine the situation on some particular road $r$ after $t_{L}$. Assume that all discontinuities on $r$ have positive speed. From the start of $r$ a single discontinuity can emerge, due to a collison with the junction along some other road connected to $r$ there. From the end of $r$ no discontinuity can emerge since this would lead to a collision between fronts which have speeds of different signs. Thus eventually all fronts on $r$ will have collided with the right endpoint of $r$ and we are left with a single virtual shock. The situation is similar if all speeds on $r$ are negative. In particular this is a well-defined construction. 
3. The Cauchy problem. In this section we will show three lemmas which then by a standard diagonalization argument imply that as $k \rightarrow \infty$ a subsequence of $\hat{z}^{k}=\left(\hat{\phi}^{k}, i_{k}\right)=$ $\Psi\left(\hat{\rho}^{k}\right)$ converges locally in $L_{1}$.

\section{Lemma 3.1.}

$$
\sup _{i, x, t}\left|z_{i}(x, t)\right| \leq 2
$$

This is obvious and needs no proof.

Now we will define the total variation of $\hat{z}^{k}$

$$
T . V .\left(\hat{z}^{k}\right)=\sum_{i=1}^{N} T . V_{\cdot x \in\left[a_{i}, b_{i}\right]} z_{i}^{k}(x, t) .
$$

Our second lemma states that the total variation is uniformly bounded in time:

\section{Lemma 3.2.}

$$
T . V \cdot\left(\hat{z}^{k}(x, t)\right) \leq K
$$

for some constant $K$ independent of $k$ and $t$.

Proof. By construction of the functional $T^{\ell}(2.20)$ and Lemma 2.3, we have that

$$
\begin{aligned}
T . V .\left(\hat{z}^{k}(x, t)\right) & \leq T^{\ell} \quad \text { for } t \in\left\langle t_{\ell-1}, t_{\ell}\right] \\
& \leq T^{1} \leq K
\end{aligned}
$$

which proves the lemma.

Lemma 3.3. Let $\tau_{1}>\tau_{2}$ be two times such that $\left(\tau_{1}-\tau_{2}\right)$ is sufficiently small. Then there is a constant $K$ independent of $k, i, \tau_{1}$ or $\tau_{2}$ such that

$$
\int_{a_{i}}^{b_{i}}\left|z_{i}^{k}\left(x, \tau_{1}\right)-z_{i}^{k}\left(x, \tau_{2}\right)\right| d x \leq K\left(\tau_{1}-\tau_{2}\right) .
$$

Proof. Let $r$ be a road with "density" $z^{k}(x, t)$ and with endpoints $a$ and $b$. Assume that $r$ is connected at $a$ through the junction $J_{a}$ with roads $r_{1}, \ldots, r_{n}$ and at $b$ through the junction $J_{b}$ with roads $r_{1+n}, \ldots, r_{m+n}$. Let $M$ be a number such that $M \geq \max \left|f^{\prime}(\rho)\right|$, such that the speed of any discontinuity in $\bar{\rho}^{k}$ is bounded by $M$. Assume now $\left(\tau_{1}-\tau_{2}\right)$ to be so small that $M\left(\tau_{1}-\tau_{2}\right) \leq \frac{1}{2} \min _{1 \leq i \leq N}\left(b_{i}-a_{i}\right)$. Define $a^{\prime}=a+M\left(\tau_{1}-\tau_{2}\right)$ and $b^{\prime}=b-M\left(\tau_{1}-\tau_{2}\right)$. For $x<a^{\prime}$, let $t_{a}(x)=\tau_{1}-\frac{x-a}{M}$, and for $x>b^{\prime}$ let $t_{b}(x)=\tau_{1}-\frac{b-x}{M}$. For $a^{\prime} \leq x \leq b^{\prime}$ we have that $\left|z_{i}^{k}\left(x, \tau_{1}\right)-z_{i}^{k}\left(x, \tau_{2}\right)\right|$ is bounded by the spatial variation of $z_{i}^{k}\left(y, \tau_{2}\right)$ where $x-M\left(\tau_{1}-\tau_{2}\right)<y<x+M\left(\tau_{1}-\tau_{2}\right)$. For $x<a^{\prime}$ or $x>b^{\prime},\left|z_{i}^{k}\left(x, \tau_{1}\right)-z_{i}^{k}\left(x, \tau_{2}\right)\right|$ is bounded by 4 by Lemma 3.1. Thus

$$
\begin{aligned}
\int_{a}^{b}\left|z^{k}\left(x, \tau_{1}\right)-z^{k}\left(x, \tau_{2}\right)\right| d x & \leq 2\left(a^{\prime}-a\right)+\int_{a^{\prime}}^{b^{\prime}}\left|z^{k}\left(x, \tau_{1}\right)-z^{k}\left(x, \tau_{2}\right)\right| d x+2\left(b-b^{\prime}\right) \\
& \leq \int_{a^{\prime}}^{b^{\prime}} \int_{x-M\left(\tau_{1}-\tau_{2}\right)}^{x+M\left(\tau_{1}-\tau_{2}\right)}\left|\frac{d z}{d y}\right| d y d x+8 M\left(\tau_{1}-\tau_{2}\right)
\end{aligned}
$$


where $\left|\frac{d z}{d y}\right|$ is a measure with total mass $T . V \cdot\left(\hat{z}^{k}\right)$. By changing the order of integration we obtain for some constant $C$

$$
\int_{a}^{b}\left|z^{k}\left(x, \tau_{1}\right)-z^{k}\left(x, \tau_{2}\right)\right| d x \leq C M\left(\tau_{1}-\tau_{2}\right) T . V \cdot\left(\hat{\rho}^{k}\right)
$$

where the right-hand side is bounded by Lemma 3.2.

Now by a standard technique, see e.g. [20, p. $385 \mathrm{ff}]$, one can show that Lemmas 3.1-3.3 imply that as $k \rightarrow \infty$, a subsequence of $\hat{z}^{k}$ converges in $L_{1}$, or more presicely

Theorem 3.1. Assume that $f$ satisfies $(\mathcal{F})$. Let $\hat{z}_{0}\left(x_{1}, \ldots, x_{N}\right)=\left(z_{1,0}\left(x_{1}\right), \ldots, z_{N, 0}\left(x_{N}\right)\right)$ be such that T.V. $\left(\hat{z}_{0}\right)$ is bounded. Then as $k \rightarrow \infty$ there exists a subsequence $k_{j}$ and a function $\hat{z}\left(x_{1}, \ldots, x_{N}, t\right)=\left(z_{1}\left(x_{1}, t\right), \ldots, z_{N}\left(x_{N}, t\right)\right)$ such that for any finite time $T$, $\hat{z}^{k_{j}}$, as defined in Sect. 2, converges uniformly to $\hat{z}$ in $L_{1}^{\text {loc }}$ for any $t<T$.

Since $\Psi^{-1}$ is uniformly continuous, this implies that some subsequence of $\hat{\rho}^{k}$ also converges to $\hat{\rho}=\Psi^{-1}(\hat{z})$.

Now we would like to show that the limit function $\hat{\rho}$ is indeed a weak solution to our problem (1.1), and to this end we have to show that the functional

$$
W(\hat{\rho})=\sum_{i=1}^{N}\left(\int_{0}^{\infty} \int_{a_{i}}^{b_{i}}\left[\rho_{i} \frac{\partial \phi_{i}}{\partial t}+f\left(\rho_{i}\right) \frac{\partial \phi_{i}}{\partial x}\right] d x d t+\int_{a_{i}}^{b_{i}} \rho_{i, 0}(x) \phi_{i}(x, 0) d x\right)
$$

vanishes for all $\phi_{i}$ in $C_{0}^{1}$ for $i=1, \ldots, N$. For simplicity we will now label our convergent subsequence $\hat{\rho}^{k}$. Since $\hat{\rho}^{k}$ almost is a weak solution of (1.2) and therefore satisfies $W\left(\hat{\rho}^{k}\right)=$ $O\left(\frac{N}{k}\right)$ with $f=f^{k}$, we can write

$$
\begin{aligned}
W(\hat{\rho})=\sum_{i=1}^{N}\left(\int _ { 0 } ^ { \infty } \int _ { a _ { i } } ^ { b _ { i } } \left[\left(\rho_{i}-\rho_{i}^{k}\right) \frac{\partial \phi_{i}}{\partial t}\right.\right. & \left.+\left(f\left(\rho_{i}\right)-f^{k}\left(\rho_{i}^{k}\right)\right) \frac{\partial \phi_{i}}{\partial x}\right] d x d t \\
& \left.+\int_{a_{i}}^{b_{i}}\left[\rho_{i, 0}(x)-\rho_{i, 0}^{k}(x)\right] \phi_{i}(x, 0) d x\right)+O\left(\frac{N}{k}\right)
\end{aligned}
$$

for any $k$. Therefore

$$
\begin{aligned}
|W(\hat{\rho})| \leq & \left\{\begin{array}{l}
\sum_{i=1}^{N}\left\|\frac{\partial \phi_{i}}{\partial t}\right\|_{\infty}\left\|\rho_{i}-\rho_{i}^{k}\right\|_{L_{1}}+\left\|\frac{\partial \phi_{i}}{\partial x}\right\|_{\infty}\left\|f\left(\rho_{i}\right)-f^{k}\left(\rho_{i}^{k}\right)\right\|_{L_{1}} \\
+\left\|\phi_{i}\right\|_{\infty}\left\|\rho_{i}(x, 0)-\rho_{i}^{k}(x, 0)\right\|_{L_{1}}
\end{array}\right. \\
\leq & \sum_{i=1}^{N}\left\|\frac{\partial \phi_{i}}{\partial t}\right\|_{\infty}\left\|\rho_{i}-\rho_{i}^{k}\right\|_{L_{1}} \\
& +\left\|\frac{\partial \phi_{i}}{\partial x}\right\|_{\infty}\left\|f\left(\rho_{i}\right)-f^{k}\left(\rho_{i}\right)\right\|_{L_{1}} \\
& +\left\|\frac{\partial \phi_{i}}{\partial x}\right\|_{\infty}\left\|f^{k}\left(\rho_{i}\right)-f^{k}\left(\rho_{i}^{k}\right)\right\|_{L_{1}} \\
& +\left\|\phi_{i}\right\|_{\infty}\left\|\rho_{i}(x, 0)-\rho_{i}^{k}(x, 0)\right\|_{L_{1}} \\
& +O\left(\frac{N}{k}\right) .
\end{aligned}
$$


By Lipshitz continuity of $f$ and $f^{k}$ the terms (3.14b) and (3.14c) are small, and the the term (3.14a) is small since $\hat{\rho}$ is the $L_{1}$ limit of $\hat{\rho}^{k}$, and the last term (3.14d) is small by the construction of $\hat{\rho}^{k}\left(x_{1}, \ldots, x_{N}, 0\right)$. Therefore for any $\epsilon>0$ we may find a $k$ such that $|W(\hat{\rho})|<\epsilon$ concluding that $\hat{\rho}$ is indeed a weak solution. Thus we have proven the following theorem.

Theorem 3.2. Consider the Cauchy problem (1.2), (1.3), (1.7) with flux function $f$ satisfying condition $(\mathcal{F})$. Assume that the initial data $\hat{\rho}_{0}\left(x_{1}, \ldots, x_{N}\right)=\left(\rho_{1}\left(x_{1}\right), \ldots, \rho_{N}\left(x_{N}\right)\right)$ is such that T.V. $\left(\Psi\left(\hat{\rho}_{0}\right)\right)$ is bounded. Then the initial boundary problem has a weak solution constructed from the solution of the Riemann problem using the entropy condition $(\mathcal{E})$.

Example Consider a simple junction consisting of two incoming roads and one outgoing. Let the density on the two incoming roads be denoted $\rho_{1}$ and $\rho_{2}$, and the density on the outgoing road be denoted $\rho_{3}$. Similarly we denote the fluxes $\phi_{i}$. Let the flux function $f$ be given by

$$
f(\rho)=4 \rho(1-\rho)
$$

and let the flux entropy function is given by $g(\phi)=f(\phi)$. Assume that we initially have the entropy state $\bar{\phi}^{\mathcal{E}}$

$$
\bar{\phi}^{\mathcal{E}}=\left(\frac{1}{3}, \frac{1}{3}, \frac{2}{3}\right) \quad \text { or } \quad \bar{\rho}=\left(\frac{3+\sqrt{6}}{6}, \frac{3+\sqrt{6}}{6}, \frac{3-\sqrt{3}}{6}\right) \text {. }
$$

Assume now that a discontinuity with left density 0 collides with the junction from road 1 at $t=0$, i.e., for negative time the density on road 1 is given by

$$
\rho_{1}= \begin{cases}0 & \text { for } x<s t \\ \frac{3+\sqrt{6}}{6} & \text { for } x \geq s t\end{cases}
$$

where $s=\frac{2}{3+\sqrt{6}}$ is the speed of the discontinuity given according to the Rankine-Hugoniot condition. Where there is no ambiguity we will also denote the densities for $t>0$ by $\rho_{i}$. After the collison the density on road 1 can only be 0 away from the junction, with a virtual shock at the endpoint connecting it to a density of 1 . Therefore the Rankine-Hugoniot condition now demands that $\phi_{2}=\phi_{3}$, and $\rho_{2}=1-\rho_{3}$. Since $g(\phi)$ has a maximum for $\phi=\frac{1}{2}$, the entropy condition $\mathcal{G}$ gives $\phi_{2}=\phi_{3}=\frac{1}{2}$, or $\rho_{2}=\frac{2+\sqrt{2}}{4}$ and $\rho_{3}=\frac{2-\sqrt{2}}{4}$. Thus we see that the densities for positive time are given by

$$
\rho_{1}(x, t)=0, \quad \rho_{2}(x, t)=\left\{\begin{array}{ll}
\frac{3+\sqrt{6}}{6} & \text { for } x<s_{2} t, \\
\frac{2+\sqrt{2}}{4} & \text { for } x \geq s_{2} t,
\end{array} \quad \rho_{3}(x, t)= \begin{cases}\frac{2-\sqrt{2}}{4} & \text { for } x<s_{3} t \\
\frac{3-\sqrt{3}}{6} & \text { for } x \geq s_{3} t .\end{cases}\right.
$$

The two speeds are given by

$$
\begin{aligned}
& s_{2}=\frac{-2}{2 \sqrt{6}-3 \sqrt{2}} \\
& s_{3}=\frac{2}{3 \sqrt{2}-2 \sqrt{3}} .
\end{aligned}
$$

Thus as expected the densities adjacent to the junction on road 2 and 3 are smaller after the collision than before. 


\section{REFERENCES}

1. D. P. Ballou, Solutions to nonlinear hyperbolic Cauchy problems without convexity conditions, Trans. Amer. Math. Soc. 152 (1970), 441-460.

2. J. H. Bick, G. F. Newell, $A$ continuum model for two-directional traffic flow, Quart. J. Appl. Math. 18 (1961), 191-204.

3. C. M. Dafermos, Polygonal approximations of solutions of the initial value problem for a conservation law, J. Math. Anal. Appl. 38 (1972), 33-41.

4. S.C. De, Kinematic wave theory of bottlenecks of varying capacity, Proc. Cambridge Phil. Soc. 52 (1956), 564-572.

5. D. C. Gazis (editor), Traffic Science, Wiley, New York, 1974.

6. H. Greenberg, An analysis of traffic flow, Oper. Res. 7 (1959), 79-85.

7. H. Holden, On the Riemann problem of a prototype of a mixed type conservation law, Comm. Pure Appl. Math. 40 (1987), 229-264.

8. H. Holden, L. Holden, On scalar conservation laws in one-dimension, in Ideas and Methods in Mathematical Analysis, Stochastics, and Applications (S. Albeverio, J. E. Fenstad, H. Holden, T. Lindstrøm, eds.), Cambridge University Press, Cambridge, 1992 (to appear).

9. H. Holden, L. Holden, R. Høegh-Krohn, A numerical method for first order nonlinear scalar conservation laws in one-dimension, Comput. Math. Applic. 15 (1988), 595-602.

10. H. Holden, L. Holden, N.H. Risebro, Some qualitative properties of $2 \times 2$ systems of conservation laws of mixed type, in Nonlinear Evolution Equations That Change Type (B. L. Keyfitz, M. Shearer, eds.), Springer, New York, 1990, pp. 67-78.

11. H. Holden, N.H. Risebro, Stochastic properties of the scalar Buckley-Leverett equation, SIAM J. Appl. Math. 51 (1991), 1472-1488.

12. H. Holden, N.H. Risebro, $A$ stochastic approach to conservation laws, in Third International Conference on Hyperbolic Problems. Theory, Numerical Methods and Applications (B. Engquist, B. Gustafsson, eds.), Studentlitteratur/Chartwell-Bratt, Lund-Bromley, 1991, pp. 575-587.

13. W. Leutzbach, Introduction to the Theory of Traffic Flow, Springer, Berlin, 1988.

14. R. J. LeVeque, Numerical Methods for Conservation Laws, Birkhäuser, Basel, 1990.

15. M. J. Lighthill, G.B. Whitham, On kinematic waves. I. Flood movement in long rivers, Proc. Roy. Soc. 229A (1955), 281-316.

16. M. J. Lighthill, G.B. Whitham, On kinematic waves. II. Theory of traffic flow on long crowded roads, Proc. Roy. Soc. 229A (1955), 317-345.

17. A. D. May, Traffic Flow Fundamentals, Prentice-Hall, Englewood Cliffs, 1990.

18. S. Mochon, An analysis of the traffic on highways with changing surface conditions, Math. Modelling 9 (1987), $1-11$.

19. P. I. Richards, Shock waves on the highway, Oper. Res. 4 (1956), 42-51.

20. J. Smoller, Shock Waves and Reaction-Diffusion Equations, Springer, New York, 1983.

21. G. B. Whitham, Linear and Nonlinear Waves, Wiley, New York, 1974.

Division of Mathematical Sciences

THE NoRWEgIan Institute of TeChNology

THE UNIVERSITY OF TRONDHEIM

N-7034 TRONDHEIM, NORWAY

E-mail: holden@imf.unit.no

Department of Mathematics

UNIVERSITY OF OSLO

P.O. BOX 1053, BLINDERN

$\mathrm{N}-0316$ OSLO, NORWAY

E-mail: nilshr@ikaros.uio.no 
